\title{
Does trans-spinal direct current stimulation modulate the Hoffmann reflexes of healthy individuals? A systematic review and meta- analysisc
}

\author{
Plínio Luna Albuquerque ${ }^{1,2,3} \cdot$ Thyciane Mendonça $^{1} \cdot$ Mayara Campêlo $^{1,2} \cdot$ Lívia Shirahige $^{1,2} \cdot$ Kátia Monte-Silva $^{1,2}$
}

Received: 11 October 2017 / Revised: 23 March 2018 / Accepted: 29 March 2018 / Published online: 12 June 2018

(c) International Spinal Cord Society 2018

\begin{abstract}
Study design Systematic review and meta-analysis.

Objectives To summarize the available evidence regarding the effects of trans-spinal direct current stimulation (tsDCS) on spinal monosynaptic circuit excitability in healthy individuals.

Setting Applied Neuroscience Laboratory, Brazil.

Methods Abstract screening was performed independently by two authors for studies found in the following databases: PubMed, CINAHL, PsycINFO, Web of Science, and LILACS. If the authors were unable to agree, a third reviewer was consulted. Randomized clinical trials that reported monosynaptic reflex measures were included. Methodological quality was assessed using the Cochrane tool for assessing the risk of bias, and information extracted about the spinal neurophysiological and stimulation protocols and their results.

Results The initial search identified 538 studies. After applying the inclusion criteria and excluding duplicates, seven crossover studies were included in the risk of bias assessment, and six studies in the meta-analyses. The meta-analysis results did not show any significant differences between anodal (pooled standardized mean difference (SMD) $=-0.09,95 \% \mathrm{CI}=$ -0.72 to $0.55, p=0.79, I^{2}=67 \%$ ) or cathodal tsDCS (pooled SMD $=0.28,95 \% \mathrm{CI}=-0.07$ to $0.63, p=0.11, I^{2}=0 \%$ ) and sham tsDCS for Hoffmann reflex modulation.

Conclusion tsDCS did not affect the Hoffmann reflex, as shown in six studies. However, these findings come from studies with selection, performance and detection bias, and further research is needed to examine the effect of this intervention.
\end{abstract}

\section{Introduction}

Spinal reflex activity plays an important role in the proper functioning of mechanisms related to tonus control and postural adjustments during movement [1]. Previous studies including injured individuals have tested therapeutic strategies, such as drug and unspecific motor interventions,

Kátia Monte-Silva

monte.silvakk@gmail.com

1 Applied Neuroscience Laboratory, Department of Physical Therapy, Universidade Federal de Pernambuco, Recife, Pernambuco, Brazil

2 Postgraduate Program in Neuropsychiatry and Behavioral Sciences, Universidade Federal de Pernambuco, Recife, Pernambuco, Brazil

3 Department of Physical Therapy, Centro Universitário Tabosa de Almeida ASCES/UNITA, Caruaru, Pernambuco, Brazil intended to increase spinal inhibitory control for post-stroke spasticity [2, 3]. However, systematic reviews have shown that motor [2,3] and pharmacological [4, 5] approaches have limited effect, and result in a risk of adverse events.

In this context, trans-spinal direct current stimulation (tsDCS) has emerged as a potential non-invasive tool for modulating spinal cord excitability and corticospinal transmission [6-8]. It involves transcutaneous application of direct current above the spinal cord over a prolonged time, to modulate physiological functions such as spinal reflexes and ascending and descending pathway transmission [9].

Previous animal model studies suggested that tsDCS exerts its effects in a polarity-dependent way. Aguilar and colleagues [7] demonstrated that anodal tsDCS reduces the local field potential responses to somatosensory stimuli in the gracile nucleus, while cathodal tsDCS does the opposite [10]. Cathodal tsDCS increases Hoffmann reflexes (Hreflexes) and spinal cord responses to cortically elicited actions [7]. 
In humans, direct current effects on spinal segmental circuitry have mainly been assessed for Ia-motoneuronal connections, by recording the Hoffmann reflex [9]. Unlike the results reported in animal studies, the effects of tsDCS on spinal monosynaptic reflexes in healthy individuals are hotly debated. Some studies found no induced changes in an H-reflex after cathodal or anodal tsDCS [11-13]; however, Lamy et al. [8] reported a leftward shift in the soleus H-reflex stimulus-response curve after anodal tsDCS (indicating increased excitability).

The H-reflex is the most often reported response in studies of spinal cord physiology [14]. The Hoffmann reflex mainly assesses the monosynaptic excitatory connection between Ia afferent from muscles spindles with alfa motoneurons $[1,14]$. This neural circuit plays a special role in the control of muscle tonus and postural adjustments during movement [1]. Deficits in Ia monosynaptic reflex inhibition contributes to the hyperexcitability of dynamic and tonic components of the stretch reflex (e.g. presynaptic and recurrent inhibition). These changes are closely related to the pathophysiology of spasticity, the most common disorder in patients with brain and spinal cord injury [15]. For this reason, understanding the effects of tsDCS on spinal monosynaptic activity in healthy individuals is important for creating clinical protocols for rehabilitation of individuals with neurological disorders like spinal cord injury and stroke, and for providing useful tools for clinical practice.

To our knowledge, no review has comprehensively evaluated the findings of available studies that assessed spinal electrophysiological measures after tsDCS in healthy individuals. Therefore, this systematic review aimed to summarize the available evidence relating to the effects of tsDCS on the excitability of spinal monosynaptic circuits in healthy individuals. We examined the acute after-effects of anodal and cathodal tsDCS on parameters of the Hoffmann reflex, comparing them to sham tsDCS.

\section{Methods}

\section{Literature search and selection criteria}

Potentially eligible articles were searched using the following databases: PubMed (via MEDLINE), CINAHL (via EBSCO), PsycINFO, Web of Science, and LILACS (via BIREME). Based on the Medical Subject Headings (MeSH), the terms used for this search were "Spinal Cord"; "Healthy Volunteers"; "Spinal Cord Stimulation"; "Humans"; and "Reflex". We used the following terms taken from the Health Sciences Descriptors (DeCS): "Healthy Volunteers" and "Reflex" in combination with MeSH terms. We also searched for the following free-text terms: "trans-spinal direct current stimulation" and "direct current stimulation". The MeSH terms, DeCS terms, and free-text terms were combined with the Boolean Operator "AND" in six combinations for all databases: "healthy volunteers" AND "spinal cord stimulation"; "spinal cord stimulation" AND reflex; "healthy volunteers" AND "transcutaneous spinal direct current stimulation"; reflex AND "transcutaneous spinal direct current stimulation"; "direct current stimulation" AND "spinal cord" and humans AND "transcutaneous spinal direct current stimulation". Additionally, we reviewed the references of included papers.

To improve the degree of specificity, we used two filters for PubMed: "Publication dates" (2008-2017) and "Species" (Humans). In all databases, "Publication dates" was used as a filter, because tsDCS was first proposed in humans in 2008. All searches were performed between February 2017 and October 2017, and language restriction was not applied. For the cases where studies were published in languages other than English, a translation was performed. The review protocol was registered in the International Prospective Register of Systematic Reviews (PROSPERO, Protocol CRD42017053810).

The research was performed following the recommendations of Cochrane Handbook for Systematic Reviews of Interventions and was described in accordance to PRISMA (Preferred Reporting Items for Systematic reviews and Meta-analysis). We included randomized controlled trials that compared spinal reflex activity through the Hoffmann reflex or through post-activation depression, after anodal or cathodal tsDCS and sham tsDCS in healthy individuals. We excluded studies that did not use a sham comparison or submitted the sham comparison to other interventions (e.g. repetitive transcranial magnetic stimulation, peripheral stimulation or behavioral interventions); and those that applied tsDCS combined with something else were thrown out.

Two reviewers (TM and PLA) analyzed the titles and abstracts of studies following the initial search in an independent way. If the authors were unable to agree, a third reviewer (MC) was consulted. After a full-text review (PLA), studies considered relevant that did not violate any exclusion criteria were retrieved for evaluation of the quality assessment phase.

\section{Quality assessment}

The methodological quality of the studies selected for the systematic review was evaluated by two independent reviewers (TM and PLA) according to chapter 8 of the Cochrane Handbook, to assess the risk of bias [16]. Each assessor performed independent assessment, then compared the results. Any differences in opinion were discussed with a third reviewer (MC). The methodological quality evaluation was used to assess random sequence generation 
Fig. 1 PRISMA flow diagram. PRISMA Preferred Reporting Items for Systematic Reviews and Meta-analyses, tDCS transcranial direct current stimulation, tsDCS trans-spinal direct current stimulation

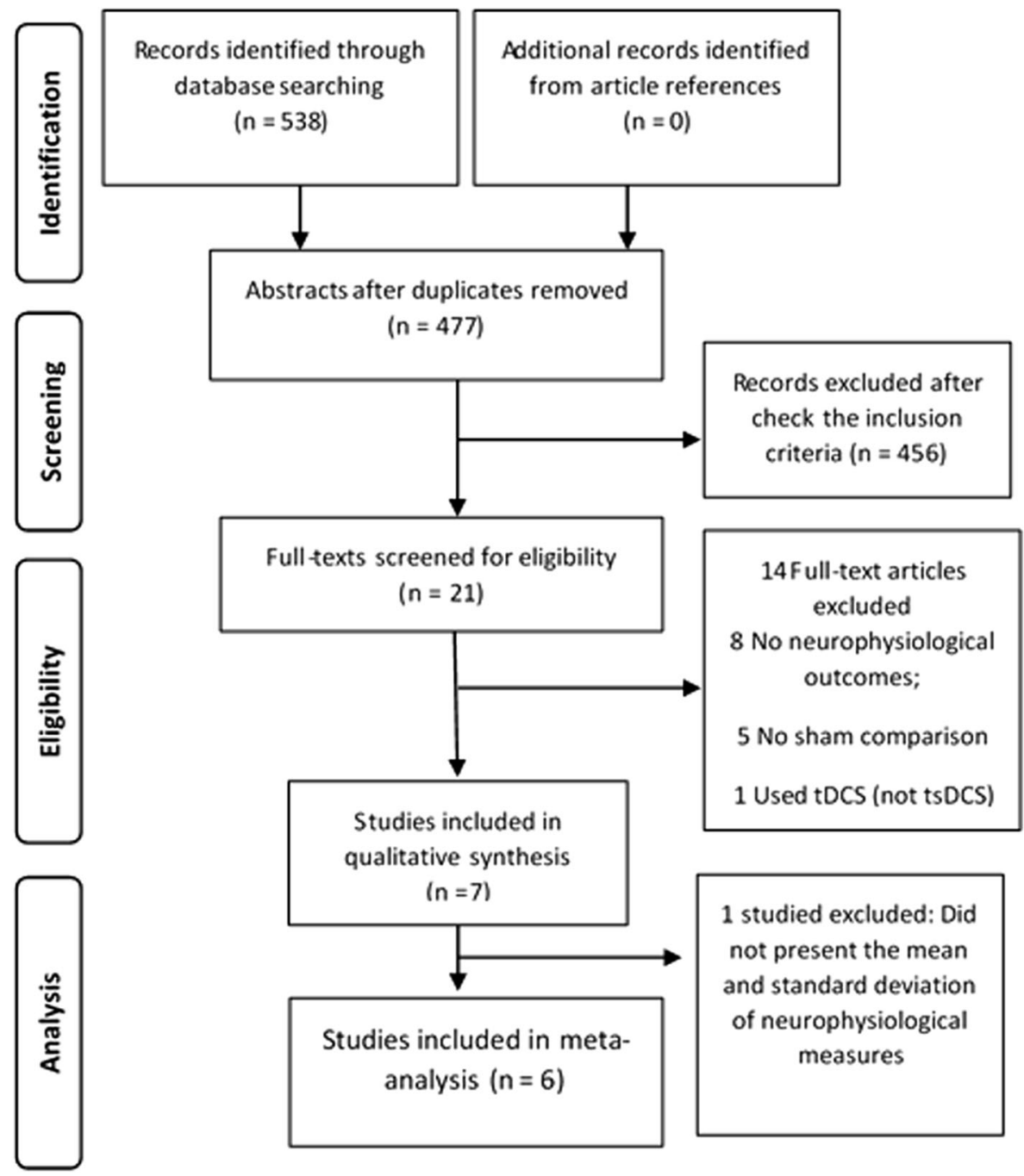

(selection bias), allocation sequence concealment (selection bias), blinding of participants (performance bias), blinding of outcome assessors (detection bias), incomplete outcome data (attrition bias), and selective reporting of outcomes (reporting bias). These biases were categorized as "low", "unclear" or "high" risk of bias [15]. The domain was considered "unclear" when not enough information to make a clear judgment was provided by the authors.

\section{Data extraction}

We created a standardized protocol to extract information considered relevant for study characterization: (i) study design; (ii) population; (iii) parameters of tsDCS; (iv) outcome measures; ( $\mathrm{v}$ ) mean \pm standard deviation (SD) of electrophysiological measures for the experimental and control sessions immediately after tsDCS.

Data selection and extraction were independently conducted at the same time by two qualified reviewers (TM and PLA). When the article did not state or did not clearly present all relevant data needed for analysis, the reviewers contacted the corresponding author. Those articles for which the author did not reply to the e-mail were excluded from the meta-analysis; only one article was excluded [17].

\section{Analysis of results}

For meta-analysis, we considered the standardized mean difference (SMD) of electrophysiological measures immediately after the intervention for the experimental sessions (anodal or cathodal tsDCS), compared to a sham tsDCS session. When studies presented results in terms of the standard error of the mean (SEM), we converted them into standard deviations (SD) using the follow equation: $\mathrm{SD}=$ $\operatorname{SEM} *(\sqrt{\mathrm{n}})$.

Review Manager Software (RevMan 5.3) was used to calculate the effect size of the difference between the spinal reflex amplitude for the sham and active tsDCS. A heterogeneity test was performed, and the studies included in the meta-analysis were considered homogeneous when the $p$ 
value was greater than 0.05 and the heterogeneity index $\left(I^{2}\right)$ was less than $25 \%$. We applied a random effects model for cases in which the heterogeneity was greater than $25 \%$, and a fixed effects model for heterogeneity indexes less than or equal to $25 \%$.

The data analysis focused on spinal cord excitability changes, measured by components of monosynaptic reflexes such as the Hoffmann reflex (e. g., the H-reflex amplitude and the $\mathrm{H}$ max/M-wave ratio) and post-activation depression. These measures were chosen because they assess spinal monosynaptic reflexes and are the most extensively reported responses in the literature to assess the spinal cord excitability. Other additional neurophysiological measures reported by the studies were described in the results table but were not included in the meta-analysis. For the meta-analysis, we used the neurophysiological recordings obtained immediately after intervention.

We selected the $\mathrm{H}$ max/M max ratio results to perform the meta-analyses, because it was a measure common to the studies included. For studies in which the H-reflex and the $\mathrm{M}$-wave amplitudes were reported separately, the $\mathrm{H} / \mathrm{M}$ ratio amplitude was calculated.

We performed two different meta-analyses, for anodal and cathodal stimulation. Two sensitivity analyses were performed to assess the robustness of the findings, since Hubli and colleagues [18] employed a different technique to assess spinal reflex activity.

\section{Results}

\section{Identification and selection of articles}

Of the 538 articles identified in the database searches, 61 were excluded as duplicates. Therefore 477 studies were selected, and 456 of these were excluded after reviewing the abstracts. Twenty-one full texts were evaluated for eligibility, of which 14 were excluded for not meeting the eligibility criteria. Seven crossover studies from various countries were carried into the risk-of-bias assessment phase (Fig. 1). One study [17] included in the quality assessment phase was excluded from the meta-analysis because it did not present the mean and standard deviation of the neurophysiological measures. Six studies were included in the meta-analyses phase, five of them in the anodal metaanalysis $[8,11-13,19]$, and five in the cathodal one $[8,11$, $13,18,19]$.

\section{Characteristics of included studies}

The six studies included in the meta-analysis were published between 2010 and 2015 [8, 11-13, 19, 20]. Altogether, 85 healthy individuals (age $25-41$ years) received

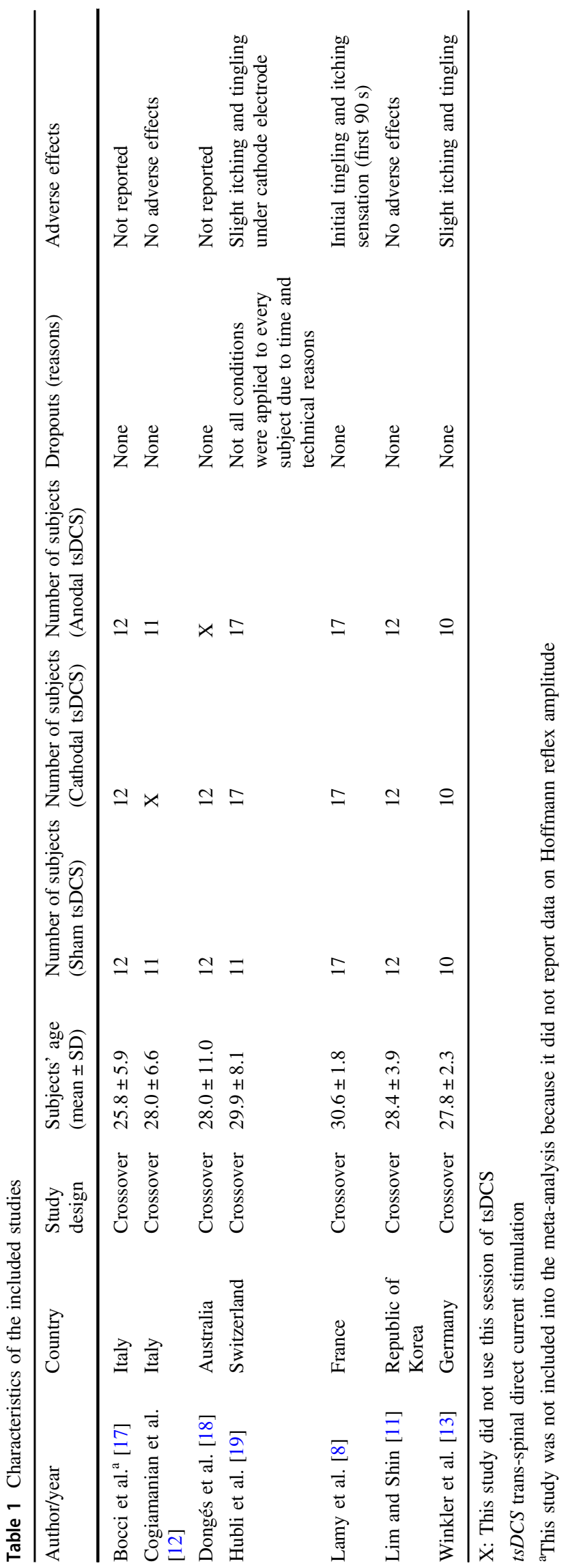


sham tsDCS, 80 of them received cathodal tsDCS, and 79 anodal tsDCS. The characteristics of the individual studies are provided in Table 1.

\section{Risk of bias assessment}

The results of the selection bias analysis showed that one study had a high risk of bias [19], while the rest were unclear or did not specify the method used to generate a random sequence $[8,11-13,17,19,20]$. With regard to allocation concealment, $57 \%$ of the included studies did not perform this methodological step [8, 11, 18, 19], while $42.86 \%$ of them failed to clearly report the method used [12, $13,20]$. Figure 2 summarizes these aspects for each of the seven studies included.

All studies failed to report on detection and performance bias. Specifically, five studies $(71.43 \%)$ did not mention blinding of the outcome assessor [11, 13, 17, 19, 20] and two (29\%) did not mention blinding of participants [11, 13] (29\%). In contrast, all studies presented a low risk of bias for attrition, reporting and other bias.

\section{tsDCS protocols and reported results}

Most studies placed the active electrode on the spinous process of the 11th thoracic vertebra and the reference electrode on the right shoulder. The most commonly used current intensity and stimulation duration were $2.5 \mathrm{~mA}$ and $15 \mathrm{~min}$, respectively. Regarding current density and total charge, the maximum values observed were $0.071 \mathrm{~mA} / \mathrm{cm}^{2}$ [8, 12, 20] and $100.8 \mathrm{mC} / \mathrm{cm}$ [19], respectively. Three studies $[8,12,13]$ assessed the electromyographic responses from the right soleus muscle, and one study from the tibialis anterior [19]. In addition, two studies recorded the Hoffmann reflex from the flexor carpi radialis [11, 20].

The $\mathrm{H} \max / \mathrm{M}$ max ratio was the neurophysiological outcome most reported by the studies [8, 11-13, 19]. In general, the studies demonstrated that $\mathrm{H}$ max/M max ratio remained unchanged after anodal, cathodal, or sham tsDCS $[8,11-13,19]$. The parameters used for tsDCS, and the main results reported by the studies, are presented in Table 2.

\section{Meta-analyses results}

In the overall meta-analyses, we failed to find a significant difference between sham tsDCS and anodal (pooled SMD $=-0.09,95 \% \mathrm{CI}=-0.72$ to $0.55, p=0.79, P^{2}=67 \%$ ) or cathodal tsDCS (pooled SMD $=0.28,95 \% \mathrm{CI}=-0.07$ to $0.63, p=0.11, I^{2}=0 \%$; Fig. 3 ). The sensitivity analyses also did not find significant differences between sham tsDCS and anodal (pooled SMD $=-0.24,95 \% \mathrm{CI}=-0.16$ to $0.63, p=0.24, \mathrm{I}^{2}=0 \%$ ) or cathodal (pooled $\mathrm{SMD}=0.12$,

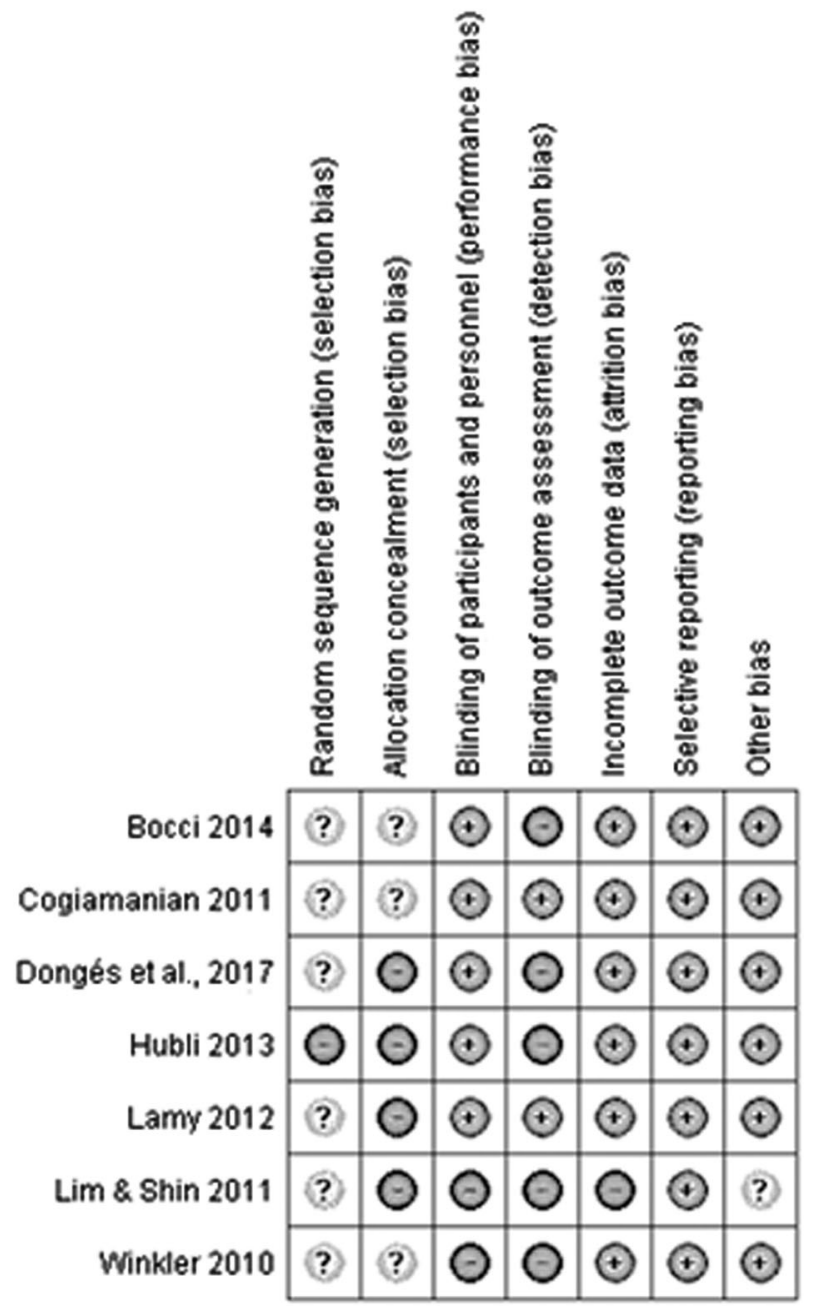

Fig. 2 Risk of bias summary: review authors' judgments about each risk-of-bias item for each included study. $(+)$ The study presented this information: "low risk of bias"; (-) the study did not report this information: "high risk of bias"; (?) uncertain bias that raises some doubt about the study's information: "unclear risk of bias". That is, the authors did not specify how this item was performed. Other bias: absence or inadequate washout time (carry-over effects in crossover studies)

95\% CI $=-0.26$ to $0.51, p=0.53, I^{2}=0 \%$ ) tsDCS. We found low heterogeneity indexes for all meta-analyses, except for the overall meta-analysis that assessed spinal excitability after anodal tsDCS $\left(p=0.02 ; I^{2}=67 \%\right)$. Table 2 summarizes the finding of other neurophysiological measures reported in the studies.

\section{Discussion}

To the best of our knowledge, this is the first systematic review and meta-analysis to assess the effects of tsDCS on monosynaptic spinal reflex excitability in healthy individuals. Our results demonstrate no difference between the 


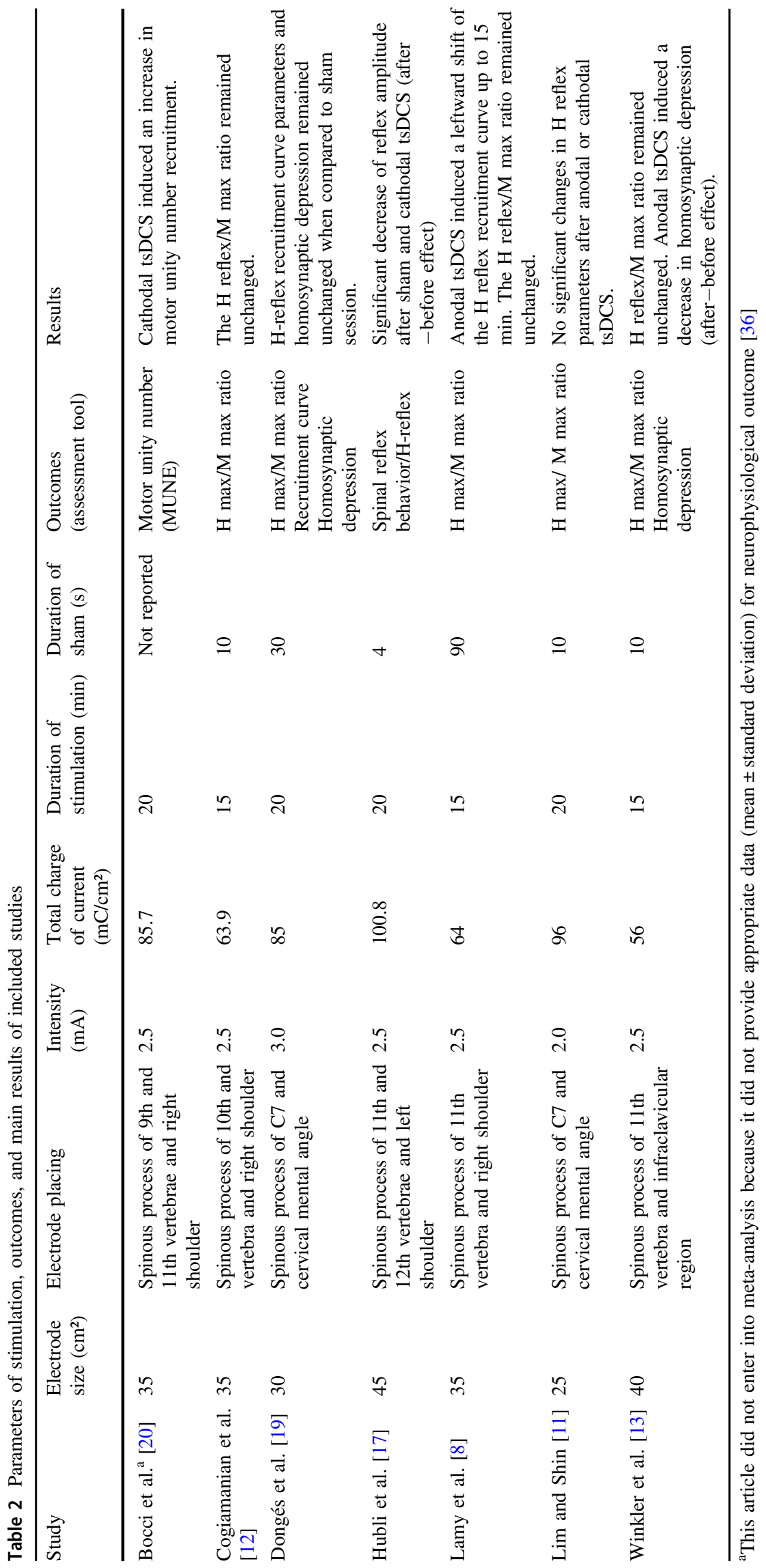




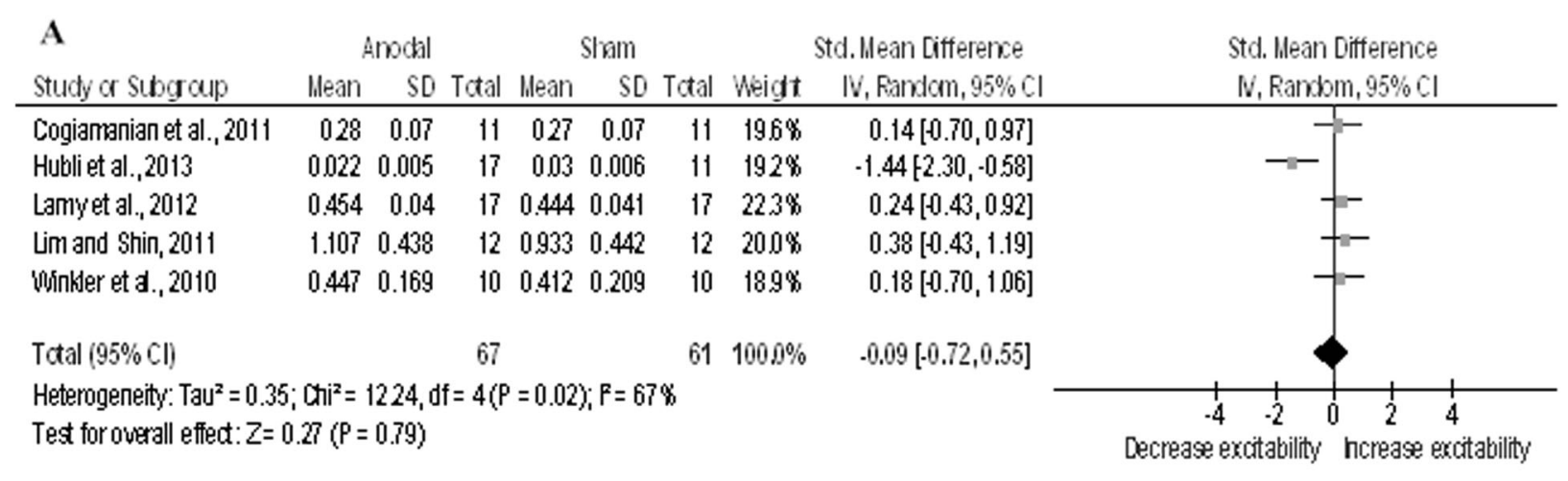

\begin{tabular}{|c|c|c|c|c|c|c|c|}
\hline \multirow{2}{*}{$\begin{array}{l}\text { B } \\
\text { Study or Subgroup }\end{array}$} & \multicolumn{3}{|c|}{ Cathodal } & \multicolumn{3}{|c|}{ Sham } & \multirow[b]{2}{*}{ Weight } \\
\hline & Wean & SD & Total & Mean & SD & Total & \\
\hline Dongés et al., 2017 & 0256 & 0.136 & 12 & 0227 & 0.143 & 12 & $190 \%$ \\
\hline Hubli et al., 2013 & 0034 & 0,008 & 17 & 0027 & 0005 & 11 & $189 \%$ \\
\hline Lamyet al., 2012 & 0.444 & 0035 & 17 & 0.444 & 0041 & 17 & 27.18 \\
\hline Lim and Shin, 2011 & 1008 & 037 & 12 & 0933 & 0.442 & 12 & $19.1 \%$ \\
\hline Uínker et al., 2010 & 0.448 & 0.169 & 10 & 0.412 & 0209 & 10 & 1598 \\
\hline \multicolumn{3}{|l|}{ Total $(95 \% \mathrm{Cl})$} & \multicolumn{3}{|l|}{68} & \multicolumn{2}{|l|}{62} \\
\hline \multicolumn{8}{|c|}{ Heterogeneity. Chi ${ }^{2}=363, \mathrm{df}=4(\mathrm{P}=0.46) ; \mathrm{F}=0 \%$} \\
\hline \multicolumn{8}{|c|}{ Test for ouerall effed: $Z=1.59(P=0.11)$} \\
\hline
\end{tabular}

Fig. 3 Forest plot of standardized mean differences of spinal cord excitability measures after trans-spinal direct current stimulation, compared to sham stimulation. a Anodal and sham trans-spinal stimulation. b Cathodal and sham trans-spinal stimulation. Mean: represents values after active and sham stimulation; $S D$ standard

values of $\mathrm{H}$ max/ $\mathrm{M}$ max amplitude ratio obtained from sham tsDCS, anodal tsDCS, or cathodal tsDCS.

The physiological mechanisms affecting the ability of tsDCS to modulate spinal cord excitability are not completely understood. However, some studies suggest that tsDCS could act by direct activation of spinal circuits, or by modulation of ascending pathways to the supra-segmental nervous system [21]. At the segmental level, tsDCS may activate inhibitory interneuron circuits [12] and thereby regulate the spontaneous activity of motor neurons [6]. Also, a probable activation of supraspinal loops, transmitted by brainstem and thalamocortical systems, has been mentioned as responsible for afferent and efferent (corticospinal tract) inhibition $[12,22]$.

Our meta-analyses show a small and nonsignificant effect size for most of the studies. Only the results of cathodal tsDCS applied by Hubli et al. [19] showed a high effect size favorable to tsDCS. Consequently, we could not confirm any changes in monosynaptic reflex excitability, which suggests that tsDCS either primarily affects neural circuits distant from the application site, or affects aspects of interneuron activity not measured by the Hoffmann reflex.

These findings differ from previous studies that assessed the effects of tsDCS in animal models. Overall, those

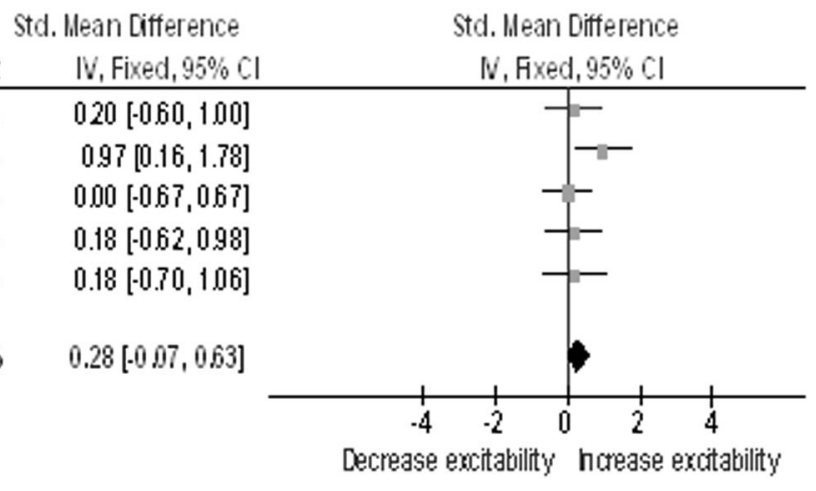

deviation; Total: indicates the number of subjects in each session. Forest plot: small squares indicate the effect size of each study; CI: 95\% confidence interval; $I^{2}$ : heterogeneity index; $\mathrm{Chi}^{2}$ : chi-square, Cochrane $\mathrm{Q}$ test. The large black diamond represents the pooled effect size of the combined trials

studies found an increase in H-reflex size after cathodal tsDCS, while anodal tsDCS had the opposite effect [7, 23]. In addition, a recent study [24] on anesthetized animals demonstrated that cathodal polarization facilitate synaptic actions evoked by peripherally stimulated afferents, including intra- and extracellularly recorded excitatory postsynaptic potentials in motoneurons. Conversely, Kaczmarek et al. found that tsDCS modulated two spinal modulatory systems related to reflex activity in a polarityindependent way, that is, cathodal and anodal tsDCS both facilitated them [25]. These studies attributed the effects of tsDCS to changes in synaptic transmission and neurotransmitter release at the spinal level.

The difference between the results presented in our metaanalysis and the results of animal studies can be partially attributed to variations in the electric field orientation relative to the spinal cord and the use of invasive electrodes. An invasive electrode arrangement generates a higher current density than that estimated for the humans who underwent tsDCS [26]. In addition, in some animals the use of anesthetic drugs can influence spinal excitability, and decreases synaptic transmission at excitatory interneuron terminals [9].

Aside from the $\mathrm{H}$ reflex/M max ratio values included in our meta-analyses, four studies presented changes in other 
spinal excitability measures [8, 12, 13, 19]. Two reported an increase in spinal excitability after anodal tsDCS through other outcomes not included in the meta-analyses, such as post-activation depression [13] and the slope of the $\mathrm{H}$ reflex recruitment curve [8]. Additionally, a reduction in the eletromyographic activity evoked by non-noxious tibial nerve stimulation during the mid-stance phase of the cycle gait was seen after cathodal tsDCS [17].

Perhaps the $\mathrm{H}$ max/M max ratio is not sensitive enough to detect spinal excitability changes induced by tsDCS, as this measure reflects a maximal percentage of depolarized motoneurons in response to Ia afferent activation. According to Lamy and colleagues [13], this measure also could be affected by external factors such as cutaneous stimulation and the duration of the experiment [8, 27]. Thus, other measures capable of assessing the transmission of the Iafiber-motoneuron synapse, such as post-activation depression, seem to be more appropriate for identifying effects induced by tsDCS [13].

All studies that reported changes in measures other than the Hoffmann reflex examined only before-and-after differences, and did not perform comparisons with sham. Since statistical differences do not prove biological causation or clinical significance, comparisons with sham groups might provide more robust conclusions about the real effect of a treatment. This could explain the absence of an effect in our meta-analyses, even if four of the seven studies included reported a spinal cord modulation after tsDCS [28]. In addition, even if it is possible that the tsDCS-induced effects were too small to differ significantly from sham stimulation, this does not mean that tsDCS has no effect on the monosynaptic reflex circuits.

Two studies contributed strongly to the meta-analyses results $[8,11]$ due a greater number of cases. The first [11] applied direct current (intensity $2.0 \mathrm{~mA}$ ) above the posterior neck in 12 volunteers. The second [8] applied direct current (intensity $2.5 \mathrm{~mA}$ ) approximately above the 11 th thoracic vertebra, in 17 volunteers. These studies used the $\mathrm{H}$ reflex to assess spinal cord excitability before and after tsDCS. In neither was sample size calculation reported. The small sample sizes employed in other studies may be responsible for failure to detect a possible existing effect [29]. We found that one study [17] involved different numbers of individuals who received active stimulation and sham stimulation. However, the authors [19] avoided multiple comparisons between the active and sham tsDCS groups, such as Friedman's test. Instead, they conducted post hoc analyses with the Wilcoxon signed rank test, thus avoiding the introduction of bias [19].

Our meta-analyses found low heterogeneity for all metaanalyses, except for anodal tsDCS, which showed moderate heterogeneity. This heterogeneity could be due to the inclusion of one study [19] that assessed H-reflex amplitude using a different method (H-reflex of the abductor hallucis longus in response to electrical stimuli consisting of an eightfold biphasic pulse with a single stimulus duration of 2 $\mathrm{ms}$ and a frequency of $200 \mathrm{~Hz}$ ). The other studies examined H-reflex values of the soleus muscle (expressed by the maximal $\mathrm{H}$ reflex/ maximal $\mathrm{M}$ wave ratio) evoked by single electrical stimulation with stimulus duration $1 \mathrm{~ms}$, delivered to the tibial nerve [8, 11-13, 18, 20]. For this reason, we performed a meta-analysis examining the standardized deviation of the mean, and a sensitivity analysis.

Regarding risk of bias, the studies included demonstrated problems with randomization sequence generation, allocation concealment, and blinding. Proper allocation concealment requires strict implementation of a random allocation sequence without foreknowledge of treatment assignments. Inadequate or unclear allocation concealment yields up to $40 \%$ larger estimates of intervention effect and contributes to an increase in heterogeneity of the results. [30] In our review, three studies $[12,13,20]$ were classified as "unclear risk of bias" and four as "high risk of bias" [8, 11, 18, 19] with respect to allocation concealment. Combined with a correct randomization process, adequate allocation sequence concealment prevents the introduction of selection bias. Neglecting these aspects indicates low methodological quality and is a serious issue.

Five studies [11, 13, 17-19] did not use blind assessors during the outcome assessment, and two [11, 13] did not apply blinding methods for participants and personnel. Blinding problems in randomized trials induce ascertainment bias and could affect the assessment of psychological or physical responses. Moreover, blinding strategies minimize the likelihood of differential treatment or assessment of outcomes [31].

Even if subjective outcomes are most at risk of detection bias, objective outcomes, including neurophysiological measures, might also involve some degree of subjectivity and thus are subject to bias [31]. We think that Hoffmann reflex measures are evaluator dependent, because factors such as electrode and stimulator positioning can modify the electromyographic results. The blinding of participants is important to ensure the same assessment conditions, since spinal reflexes can be increased when individuals are highly aroused, anxious, or in a fearful state [32, 33]. Prior knowledge of the type of stimulation (real or sham) could increase the level of anxiety of individuals and induce changes in electromyographic responses unrelated to tsDCS. Inadequate allocation concealment can also affect the compliance and retention of trial participants [34].

It is relevant to consider that the studies included adopted very similar protocols for tsDCS. In general, the protocols used a current intensity from 2.0 to $2.5 \mathrm{~mA}$ and a stimulation duration ranging from 15 to $20 \mathrm{~min}$. These parameters are considered safe and far below to the threshold for tissue damage; in addition, no serious adverse effects were reported [26, 35].

This systematic review represents an important guide for future studies in the neurophysiology field that aim 
to evaluate the effects of tsDCS on spinal monosynaptic circuits. Our meta-analysis found no differences between the effects produced by sham tsDCS and anodal or cathodal tsDCS, in their ability to modulate spinal excitability of healthy individuals, as assessed by the H-reflex (H-reflex/M max ratio). However, these results involve studies with selection, performance, and detection bias.

The changes induced by tsDCS may not have a significant influence on the excitability of alpha-motoneurons, but may affect the efficacy of Ia fiber-motoneuron synapse, or they could regulate the activity of the inhibitory interneuron network. Neither of these effects are fully evaluated by the Hoffmann reflex, and therefore could be missed; thus, additional measures such as post-activation depression should be included in future studies to complement the results from Hoffmann reflex analysis.

Our results suggest that tsDCS causes no change in a physiological parameter in healthy individuals. However, further studies should examine the effect of tsDCS on polysynaptic circuits and neurotransmitter release at the spinal cord level. These studies should employ a larger sample size and greater methodological rigor, especially concerning allocation concealment and randomization.

\section{Data availability}

All relevant data are provided within the paper.

\begin{abstract}
Author contributions PLA was responsible for designing the review protocol, writing the protocol and report, conducting the search, screening potentially eligible studies, extracting and analyzing data, interpreting results, draft and revising the manuscript. TM was responsible for conducting the search, screening potentially eligible studies and extracting data, updating reference lists and creating tables. MC was responsible for designing the review protocol, judging possible disagreements during the article's search and creating tables. LS was responsible for analyzing data, graphic generation and interpreting results. KMS was responsible for overall supervision, revising the manuscript and approving the final version.
\end{abstract}

Funding Katia Monte-Silva is supported by the Conselho Nacional de Desenvolvimento Científico e Tecnológico (CNPQ), Brazil (308291/ 2015-8).

\section{Compliance with ethical standards}

Conflict of interest The authors declare that they have no conflict of interest.

\section{References}

1. Jankowska E. Spinal interneuronal systems: identification, multifunctional character and reconfigurations in mammals. J Physiol. 2001;533:31-40.
2. Corbetta D, Sirtori V, Castellini G, Moja L, Gatti R. Constraintinduced movement therapy for upper extremities in people with stroke. Cochrane Database Syst Rev. 2015;8:1-116.

3. Kinnear BZ, Lannin NA, Cusick A, Harvey LA, Rawicki B. Rehabilitation therapies after botulinum toxin-A injection to manage limb spasticity: a systematic review. Phys Ther. 2014;94:1569.

4. Lindsay C, Kouzouna A, Simcox C, Pandyan AD. Pharmacological interventions other than botulinum toxin for spasticity after stroke. Cochrane Database Syst Rev. 2016;10:1-63.

5. McIntyre A, Lee T, Janzen S, Mays R, Mehta S, Teasell R. Systematic review of the effectiveness of pharmacological interventions in the treatment of spasticity of the hemiparetic lower extremity more than six months post stroke. Top Stroke Rehabil. 2012;19:479-90.

6. Ahmed Z. Trans-spinal direct current stimulation modulates motor cortex-induced muscle contraction in mice. J Appl Physiol. 2011;110:1414-24.

7. Ahmed Z, Wieraszko A. Trans-spinal direct current enhances corticospinal output and stimulation-evoked release of glutamate analog, D-2, 3-3H-aspartic acid. J Appl Physiol. 2012;112:1576-92.

8. Lamy J-C, Ho C, Badel A, Arrigo RT, Boakye M. Modulation of soleus $\mathrm{H}$ reflex by spinal DC stimulation in humans. J Neurophysiol. 2012;108:906-14.

9. Priori A, Ciocca M, Parazzini M, Vergari M, Ferrucci R. Transcranial cerebellar direct current stimulation and transcutaneous spinal cord direct current stimulation as innovative tools for neuroscientists. J Physiol. 2014;592:3345-69.

10. Aguilar J, Pulecchi F, Dilena R, Oliviero A, Priori A, Foffani G. Spinal direct current stimulation modulates the activity of gracile nucleus and primary somatosensory cortex in anaesthetized rats. J Physiol. 2011;589:4981-96.

11. Lim C-Y, Shin H-I. Noninvasive DC stimulation on neck changes MEP. Neuroreport . 2011;22:819-23.

12. Cogiamanian F, Vergari M, Schiaffi E, Marceglia S, Ardolino G, Barbieri S, et al. Transcutaneous spinal cord direct current stimulation inhibits the lower limb nociceptive flexion reflex in human beings. Pain . 2011;152:370-5.

13. Winkler T, Hering P, Straube A. Spinal DC stimulation in humans modulates post-activation depression of the H-reflex depending on current polarity. Clin Neurophysiol. 2010;121:957-61.

14. Misiaszek JE. The H-reflex as a tool in neurophysiology: its limitations and uses in understanding nervous system function. Muscle Nerve. 2003;28:144-60.

15. Nielsen JB, Crone C, Hultborn H. The spinal pathophysiology of spasticity-from a basic science point of view. Acta Physiol. 2007;189:171-80.

16. Higgins J, Altman D, Sterne J. Chapter 8: Assessing risk of bias in included studies. Cochrane Handbook for Systematic Reviews of Interventions Version 5.1. 0 [updated March 2011]. Cochrane Database Syst Rev. 2011;5:187-241.

17. Bocci T, Vannini B, Torzini A, Mazzatenta A, Vergari M, Cogiamanian F, et al. Cathodal transcutaneous spinal direct current stimulation (tsDCS) improves motor unit recruitment in healthy subjects. Neurosci Lett. 2014;578:75-9.

18. Dongés SC, D'Amico JM, Butler JE, Taylor JL. The effects of cervical transcutaneous spinal direct current stimulation on motor pathways supplying the upper limb in humans. PLoS ONE. 2017;12:e172333.

19. Hubli M, Dietz V, Schrafl-Altermatt M, Bolliger M. Modulation of spinal neuronal excitability by spinal direct currents and locomotion after spinal cord injury. Clin Neurophysiol. 2013;124:1187-95.

20. Cohen J. Statistical power analysis. Curr Dir Psychol Sci. 1992;1:98-101. 
21. Grecco LH. Transcutaneous spinal stimulation as a therapeutic strategy for spinal cord injury: state of the art. J Neurorestoratology. 2015;3:73-82.

22. Cogiamanian F, Ardolino G, Vergari M, Ferrucci R, Ciocca M, Scelzo E, et al. Transcutaneous spinal direct current stimulation. Front Psychiatry. 2012;3:1-5.

23. Ahmed Z. Effects of cathodal trans-spinal direct current stimulation on mouse spinal network and complex multijoint movements. J Neurosci. 2013;33:14949-57.

24. Bolzoni F, Jankowska E. Presynaptic and postsynaptic effects of local cathodal DC polarization within the spinal cord in anaesthetized animal preparations. J Physiol. 2015;593:947-66.

25. Kaczmarek D, Ristikankare J, Jankowska E. Does trans-spinal and local DC polarization affect presynaptic inhibition and post-activation depression? J Physiol. 2017;595:1743-61.

26. Parazzini M, Fiocchi S, Liorni I, Rossi E, Cogiamanian F, Vergari $\mathrm{M}$, et al. Modeling the current density generated by transcutaneous spinal direct current stimulation (tsDCS). Clin Neurophysiol. 2014;125:2260-70.

27. Lagerquist $\mathrm{O}$, Collins DF. Influence of stimulus pulse width on Mwaves, H-reflexes, and torque during tetanic low-intensity neuromuscular stimulation. Muscle Nerve. 2010;42:886-93.
28. Skelly AC. Probability, proof, and clinical significance. Evid Based Spine Care J. 2011;2:9-11.

29. Noordzij M, Tripepi G, Dekker FW, Zoccali C, Tanck MW, Jager KJ. Sample size calculations: basic principles and common pitfalls. Nephrol Dial Transplant. 2010;25:1388-93.

30. Schulz KF, Grimes DA. Allocation concealment in randomised trials: defending against deciphering. Lancet . 2002;359:614-8.

31. Karanicolas PJ, Farrokhyar F, Bhandari M. Blinding: who, what, when, why, how? Can J Surg. 2010;53:345.

32. Rosenbaum DA. Chapter 3-Physiological foundations. Human motor control. 2nd ed. San Diego: Academic Press; 2010. p. 43-91.

33. Sibley KM, Carpenter MG, Perry JC, Frank JS. Effects of postural anxiety on the soleus H-reflex. Hum Mov Sci. 2007;26:103-12.

34. Schulz KF, Grimes DA. Blinding in randomised trials: hiding who got what. Lancet . 2002;359:696-700.

35. Bikson M, Grossman P, Thomas C, Zannou AL, Jiang J, Adnan T, et al. Safety of transcranial direct current stimulation: evidence based update 2016. Brain Stimul. 2016;9:641-61.

36. Hubli M, Dietz V, Bolliger M. Spinal reflex activity: a marker for neuronal functionality after spinal cord injury. Neurorehabil Neural Repair. 2012;26:188-96. 